\title{
Diagnósticos e intervenções de enfermagem frequentes em mulheres internadas em uma unidade de terapia intensiva*
}

\author{
Frequent nursing diagnoses and interventions for women under critical care
}

Diagnósticos y intervenciones de enfermería frecuentes en mujeres en una unidad de terapía intensiva

\author{
Mirna Fontenele de Oliveira', Maria Célia de Freitas' \\ 'Universidade Estadual do Ceará. Departamento de Enfermagem, Mestrado em Cuidados Clínicos. Fortaleza, CE
}

Submissão: $12 / 05 / 2008$

Aprovação: 2 l/03/2009

\section{RESUMO}

Objetivou-se identificar os Diagnósticos de Enfermagem mais freeüentes e propor intervenções para mulheres internadas em uma unidade de terapia intensiva materna de um hospital público de Fortaleza-CE. Estudo retrospectivo, realizado em prontuários de mulheres internadas. Foram elaborados dez Diagnósticos de Enfermagem. Destes, Quatro de risco: risco para infecção, risco para deseQuilíbrio no volume de lí́uidos, risco para aspiração, risco para integridade da pele prejudicada e seis reais: maternidade alterada, mobilidade física prejudicada, ansiedade e comunicação verbal prejudicada. Para os referidos Diagnósticos de Enfermagem, propõem-se intervenções, segundo a ligação entre NANDA, NIC e NOC. Conclui-se que o uso dos Diagnósticos de Enfermagem é tecnologia necessária ao cotidiano de enfermagem, pois possibilita o cuidado integral.

Descritores: Diagnóstico de Enfermagem; Cuidados intensivos; Saúde da mulher.

\begin{abstract}
This study aimed at identifying the most frequent Nursing Diagnoses and propose interventions for women under critical care into a maternal intensive care unit in a public hospital in Fortaleza, CE, Brazil. Retrospective study conducted with women's hospital health records. Ten Nursing Diagnoses were elaborated, being four risk: risk of infection, risk of unbalance of lieuids volume, risk of aspiration, risk of damaged skin integrity and six real: altered maternity, impaired physical mobility, anxiety and impaired verbal communication. For the referred Nursing Diagnoses, nursing interventions are proposed according to the link between NANDA, NIC and NOC. It was concluded that the use of ND is a necessary technology for the Nursing reality, as it makes possible the integral care.
\end{abstract}

Descriptors: Nursing diagnosis; Intensive care; Women's health.

\section{RESUMEN}

El objetivo del estudio fue identificar los Diagnósticos de Enfermería más frecuentes y proponer intervenciones para mujeres internadas en una unidad de terapia intensiva maternal de un hospital público en Fortaleza, CE, Brasil. Estudio retrospectivo realizado con los prontuarios de mujeres internadas. Se elaboró diez Diagnósticos de Enfermería, cuatro de riesgo: riesgo para infección, riesgo para desequilibrio en el volumen de líquidos, riesgo para aspiración, riesgo para integridad de la piel perjudicada y seis reales: maternidad alterada, mobilidad física perjudicada, ansiedad y comunicación verbal perjudicada. Para los referidos DE, se propone intervenciones, según la ligación entre NANDA, NIC y NOC. Se concluye que el uso de los DE es una tecnología necesaria a la rutina de la enfermería, ya Que posibilita la atención integral.

Descriptores: Diagnóstico de enfermería; Cuidados intensivos; Salud de la mujer.

* Artigo extraído da monografia de Especialização em Unidade de Terapia Intensiva apresentada à Universidade Estadual do Ceará, Fortaleza-CE, intitulada: "Diagnósticos e intervençôes de enfermagem freQüentes nas pacientes de uma unidade de terapia intensiva materna". 


\section{INTRODUÇÃO}

O termo enfermagem obstétrica origina-se da prática clínica no planejar cuidados de enfermagem à gestante, desde a concepção até seis semanas após o parto. Dessa forma, a enfermeira deve conhecer o desenvolvimento humano e facilitar um ambiente Que ajude os indivíduos a se desenvolverem cognitivamente, psicossocialmente e fisicamente, sobretudo no momento da gravidez e do puerpério.

Embora a gravidez seja um evento biologicamente normal, alguns fatores podem estar presentes de forma a tornarem-se riscos potenciais de complicação para a saúde da mãe e/ou filho. Identificar o limite entre a normalidade e a doença instalada é fundamental na prevenção da morbi-mortalidade materno-fetal. Nesse sentido, considera-se como mortalidade materna o óbito da mulher grávida, ou dentro de 42 dias completos de puerpério, independentemente da duração da prenhez e do local de implantação do ovo, mas extremamente relacionado a causas associadas, agravos desencadeados pela gestação e ao tratamento recebido ${ }^{(1)}$.

As complicações obstétricas são responsáveis por Quase 50\% de todas as mortes maternas no mundo, e o risco de morrer é até 25 vezes maior em países subdesenvolvidos, como o Brasil, Quando comparados aos países desenvolvidos ${ }^{(1)}$.

Quando as complicações obstétricas não são tratadas em tempo hábil, poderão evoluir para um agravo clínico Que necessite de tratamento crítico, no caso, em uma Unidade de Terapia Intensiva (UTI). Lá, boa parte dessas mulheres serão cuidadas na tentativa de reverter as instabilidades clínicas, oferecendo condições de recuperação e reintegração social.

Diante desta situação, os enfermeiros das Unidades de Terapia Intensiva tornar-se-ão peça fundamental no tratamento e recuperação dessas mulheres. Com esta finalidade, eles deverão ter amplo conhecimento científico sobre patologias comuns da gestação e puerpério, para prestar assistência às suas clientes, utilizando como referencial o DE. Este diagnóstico permite o planejamento do cuidado fundamentado em conhecimentos científicos e utilização do raciocínio clínico.

Tais reQuisitos são considerados indispensáveis ao se considerar a enfermagem como a ciência e a arte de assistir o ser humano no atendimento de suas necessidades humanas básicas, de torná-lo independente desta assistência Quando possível, pelo ensino do autocuidado; de recuperar, manter, promover a saúde em colaboração com outros profissionais ${ }^{(2)}$.

Em um ambiente de UTI as necessidades de cuidado de enfermagem aumentam, pois os clientes irão precisar de avaliações críticas e rápidas, planos de cuidados abrangentes, serviços bem coordenados com outros profissionais do cuidado da saúde, além de um efetivo e conveniente planejamento de alta ${ }^{(3)}$.

Portanto, a utilização das etapas do processo de enfermagem apresenta-se como ferramenta válida na melhoria da assistência de enfermagem, nesse caso, em Unidades de Terapia Intensiva Materna.

Continuamente, as etapas do processo de enfermagem visam atualizar as informações da equipe de enfermagem sobre o estado de saúde do paciente, de forma a planejar em detalhes a relação entre o paciente e a enfermeira responsável pela assistência, e assim, tornar o cuidado mais humano e eficaz ${ }^{(4)}$.

Como mostra a experiência, a utilização do processo de assistência em enfermagem vem garantir a prestação de um atendimento individualizado, segundo as necessidades da clientela.

Em face da essência do papel da equipe de enfermagem no cuidado a mulher com complicações obstétricas ou ginecológicas, e por reconhecer a existência do predomínio de atividades técnicas, Que se distanciam do enfoque humanístico, resolveu-se realizar este estudo, cujo objetivo foi o seguinte: identificar os Diagnósticos e Intervenções de Enfermagem mais freqüentes nas mulheres internadas em uma UTI Materna, juntamente com os fatores relacionados e características definidoras, segundo a taxonomia I da NANDA, como possibilidade de sensibilizar a equipe para a utilização do Processo de Enfermagem no cotidiano.

Tal estudo torna-se relevante à medida Que fornece dados teóricopráticos sobre a importância da aplicabilidade do Processo de Enfermagem, contribuindo para reflexões futuras voltadas à melhoria da assistência de enfermagem em Unidades de Terapia Intensiva Materna.

\section{METODOLOGIA}

Desenho não-experimental com tipo de pesQuisa inter-relacional retrospectiva com base em prontuários de mulheres internadas em uma UTI Materna no período de janeiro a junho de 2005. Para a referida análise, foram observadas as anotações e evoluções tanto de enfermagem, como médicas das pacientes internadas.

A pesquisa foi desenvolvida em uma maternidade-escola da rede pública, referência no atendimento materno-infantil da cidade de Fortaleza-CE, onde está localizada uma Unidade de Terapia Intensiva específica para o atendimento de complicações gineco-obstétricas. O levantamento dos dados deu-se no segundo semestre de 2005.

Quanto à coleta de dados, foi feita em dois momentos. No primeiro, procedeu-se a uma análise retrospectiva nos prontuários de todas as mulheres Que estiveram internadas na UTI, durante o primeiro semestre de 2005. Buscou-se, inicialmente, aplicar um formulário pré-elaborado com dados de identificação e da história de saúde das pacientes, a fim de obter dados que contribuíssem para a elaboração de um julgamento clínico. De acordo com a literatura, a história de saúde oferece indicações sobre problemas atuais ou potenciais, culturais e socioeconômicos úteis para a elaboração de um plano de assistência eficaz ${ }^{(4)}$.

No segundo momento, investigaram-se nas evoluções e/ou anotações tanto de enfermagem, como médicas existentes nos prontuários, dados Que complementassem e auxiliassem na elaboração do julgamento clínico, rumo à identificação das inferências diagnósticas e, posteriormente, confirmação dos Diagnósticos de Enfermagem nelas presentes. Ressalta-se Que a construção do raciocínio Diagnóstico de Enfermagem foi fundamentada nos parâmetros propostos por Risner ${ }^{(5)}$.

O raciocínio diagnóstico é um processo Que possibilita a um observador atribuir um significado e classificar um fenômeno em situação clínica, pela integração da observação e pensamento crítico ${ }^{(6)}$.

Assim, foi possível a elaboração dos Diagnósticos de Enfermagem, conforme a Taxonomia I da North American Nursing Diagnosis Association (NANDA) ${ }^{(7)}$.

Buscou-se, ainda, a validação dos Diagnósticos e Intervenções de Enfermagem encontrados, junto às enfermeiras assistenciais da UTI Materna, mediante um impresso tipo cheque-lista, no Qual 
constavam os Diagnósticos de Enfermagem e suas respectivas propostas de intervenções, segundo a Nursing Interventions Classification $(\mathrm{NIC})^{(8)}$, Que foram preenchidas pelas enfermeiras. Após, foi realizado um comparativo entre as intervenções propostas com aQuelas executadas pelas enfermeiras.

Para realizar a validação, as enfermeiras foram buscadas em seus diferentes turnos de trabalho: manhã, tarde e noite, excluindose apenas aquelas que estiveram ausentes por férias, licença médica ou licença gestante.

Contou-se com a colaboração de dez enfermeiras, funcionárias da unidade, interessadas em participar e contribuir com o estudo, após prévia exposição dos objetivos e assinatura do termo de consentimento livre e esclarecido.

De posse de uma população de 106 prontuários, fez-se uma média do período de internamento, e encontrou-se uma média de três dias de internamento. Deste, extraiu-se uma amostra de 28 prontuários, os Quais tiveram como critério de inclusão um período de internamento igual ou superior à média (três dias).

Por se tratar de uma análise documental, respeitaram-se os preceitos éticos e legais, conforme a resolução 196/96 ${ }^{(9)}$. Como exigido, houve assinatura do termo de fiel depositário, o envio do projeto da pesquisa para avaliação institucional e posterior consentimento para consulta nos prontuários.

Fez-se, ainda, o encaminhamento do projeto ao Comitê de Ética em Pesquisa da Universidade Federal do Ceará, para apreciação e aprovação.

Cumpridos estes procedimentos, adotou-se uma abordagem Qualitativa, sendo os dados analisados após serem agrupados segundo suas convergências, com discussão fundamentada em teóricos estudiosos do assunto.

\section{RESULTADOS E DISCUSSÃO}

Do total das mulheres pesquisadas, a maioria possuía entre 20 e 29 anos (40\%), com nível básico de escolaridade (5 I \% estudaram até o $I^{\circ}$ grau) e um percentual de duas a Quatro gestações.

Para a segunda etapa do estudo, em Que se procederá ao julgamento clínico dos problemas de enfermagem, para posterior elaboração dos Diagnósticos de Enfermagem. Foram selecionadas apenas aquelas pacientes Que permaneceram na UTI Materna por um número de dias maior ou igual à média encontrada, três dias, perfazendo um total de 28 mulheres (29\% do total).

Entre os motivos de internamento na UTI Materna, encontraramse: pré-eclâmpsia grave $(28,5 \%)$, HELLP síndrome $(21,4 \%)$, eclâmpsia $(17,9 \%)$, distúrbios hematológicos $(10,7 \%)$, distúrbios endócrinos $(7,1 \%)$, abortamento retido $(3,6 \%)$, deslocamento prematuro de placenta $(3,6 \%)$, intoxicação exógena $(3,6 \%)$ e distúrbios eletrolíticos $(3,6 \%)$.

Mediante análise dos dados da história de saúde/doença das mulheres, juntamente com os registros médicos e de enfermagem, procedeu-se ao julgamento clínico, identificando-se os Diagnósticos de Enfermagem, os Quais foram descritos conforme Taxonomia I da $\operatorname{NANDA}^{(7)}$.

Buscou-se também comparar as intervenções propostas pela Nursing Interventions Classification, descritos na ligação entre NANDA, NIC E NOC (8), com as realizadas pelas enfermeiras assistenciais da UTI materna. A NIC consta de uma classificação completa, padronizada, das intervenções propostas aos enfermeiros.

Após identificados, os DE foram listados em um formulário, tipo cheque-lista, juntamente com as principais intervenções propostas pela NIC. Estes foram entregues às enfermeiras assistenciais da UTI para etapa de validação.

Contou-se com a participação de dez enfermeiras, Que preencheram o formulário proposto e validaram o achado, tornando a peseuisa mais fidedigna.

Foram identificados dez Diagnósticos de Enfermagem agrupados conforme seu padrão de resposta humana. Cinco deles correspondem ao padrão de resposta TROCAR, dois ao padrão de resposta RELACIONAR, um ao padrão de resposta MOVER, um ao padrão de resposta SENTIR, um ao padrão de resposta COMUNICAR (Quadro 1); e Quatro deles são de risco. Isto demonstra que as pacientes possuíam déficits em necessidades humanas bem diversificadas.

De acordo como o Quadro I as freqüências dos diagnósticos, características definidoras e fatores de risco do padrão de respostas humanas TROCAR. Os diagnósticos mais freqüentes foram: Risco para infecção (presente em todos os casos), Risco para desequilíbrio no volume de líquidos ( 4 casos), Risco para integridade da pele prejudicada (presente em todos os cacos), Risco para aspiração (16 casos), Padrão respiratório ineficaz ( 20 casos).

Segundo observou-se, os diagnósticos deste padrão tiveram a maior freeüência, englobando todos os diagnósticos de risco, demonstrando eue a gravidade das pacientes pesquisadas lhes configurava uma alta vulnerabilidade a potenciais complicações. Em uma Unidade de Terapia Intensiva encontram-se pacientes em risco de vida. Cabe ao enfermeiro reconhecer os sinais iniciais de desvio da normalidade para implementar uma assistência de enfermagem de Qualidade ${ }^{(10)}$.

Das principais intervenções propostas pela NIC para o diagnóstico de enfermagem Risco para infecção: cuidado com lesões; cuidado com local de incisão; cuidado na manipulação de sondas, drenos e cateteres; lavagem das mãos e prevenção de infecção cruzada, todas foram checadas pelas enfermeiras assistenciais da UTI Materna.

No contexto da assistência de enfermagem em UTI, onde os pacientes são de maior risco, especialmente por serem submetidos à procedimentos invasivos freQüentes ou à administração de antibióticos de amplo espectro, oportunizando a seleção de microrganismos resistentes, a atenção às medidas preventivas reveste-se de redobrado significado( ${ }^{(1)}$.

Das principais intervenções propostas pela NIC para o diagnóstico de enfermagem Risco para o desequilíbrio no volume de líquidos: controle de líquidos e monitorização de líquidos, todas foram checadas pelas enfermeiras assistenciais da UTI Materna. Em um estudo realizado em 2003, o DE Risco para desequilíbrio no volume de líquido também esteve presente em 100\% das pacientes internadas em UTI ${ }^{(12)}$

Das principais intervenções propostas pela NIC para o diagnóstico de enfermagem Risco para integridade da pele prejudicada: cuidados com pele e mucosas e mudança de decúbito, todas foram checadas pelas enfermeiras assistenciais da UTI Materna. Conforme se percebe, é muito comum o surgimento de feridas em pacientes de longa permanência em leitos de UTI, em decorrência, sobretudo, da imobilidade física, com vasoconstrição periférica e diminuição da oxigenação tecidual. 
Quadro I. Diagnósticos de Enfermagem segundo os padrões da Taxonomia I da NANDA, encontrados em mulheres sob terapia intensiva. Fortaleza, CE, 2005.

\begin{tabular}{|c|c|c|}
\hline $\begin{array}{l}\text { DIAGNÓSTICOS DE } \\
\text { ENFERMAGEM }\end{array}$ & $\begin{array}{c}\text { CARACTERISTICAS DEFINIDORAS } \\
\text { FATORES DE RISCO OU FATORES RELACIONADOS }\end{array}$ & $f i$ \\
\hline \multicolumn{3}{|l|}{ Padrão Trocar } \\
\hline Risco para infecção & $\begin{array}{c}\text { Procedimento invasivo } \\
\text { Exposição a flora patológica da UTI } \\
\text { Imunossupressão } \\
\text { Desnutrição } \\
\text { Doença crônica }\end{array}$ & 28 \\
\hline $\begin{array}{l}\text { Risco para desequilíbrio no } \\
\text { volume de líquidos }\end{array}$ & Restrição ao leito & 14 \\
\hline $\begin{array}{l}\text { Risco para integridade da pele } \\
\text { prejudicada }\end{array}$ & $\begin{array}{c}\text { Imobilidade física } \\
\text { Estado metabólico alterado } \\
\text { Desnutrição }\end{array}$ & 28 \\
\hline Risco para aspiração & $\begin{array}{c}\text { Alimentação por sondas } \\
\text { Nível de consciência reduzido } \\
\text { Presença de traQueostomia ou tubo endotraqueal } \\
\text { Resíduo gástrico aumentado } \\
\text { Esvaziamento gástrico retardado }\end{array}$ & 16 \\
\hline Padrão respiratório ineficaz & $\begin{array}{l}\text { Ventilação minuto irregular } \\
\text { Dispnéia } \\
\text { Excursão torácica aumentada }\end{array}$ & 20 \\
\hline \multicolumn{3}{|l|}{ Padrão Relacionar } \\
\hline Interação social prejudicada & $\begin{array}{l}\text { Inabilidade verbalizada ou observada para receber ou comunicar uma } \\
\text { sensação } \\
\text { Interação disfuncional com pessoas da família ou profissionais de saúde } \\
\text { Isolamento terapêutico, mobilidade física prejudicada, barreiras ambientais, } \\
\text { ausência de pessoas significativas }\end{array}$ & 28 \\
\hline Maternidade alterada & $\begin{array}{c}\text { Déficit na interação mãe-filho } \\
\text { Doença física }\end{array}$ & 28 \\
\hline \multicolumn{3}{|l|}{ Padrão Mover } \\
\hline Mobilidade física prejudicada & $\begin{array}{l}\text { Dificuldade para virar-se } \\
\text { Restrição de movimentos prescritos } \\
\text { Amplitude limitada de movimentos }\end{array}$ & 28 \\
\hline \multicolumn{3}{|l|}{ Padrão Sentir } \\
\hline Ansiedade & $\begin{array}{l}\text { Agitação,ineuietação, irritabilidade,angústia } \\
\text { Ameaça de mudança no status do papel } \\
\text { Ameaça ou mudança no estado de saúde }\end{array}$ & 28 \\
\hline \multicolumn{3}{|l|}{ Padrão Comunicar } \\
\hline Comunicação verbal prejudicada & $\begin{array}{c}\text { Fala ou verbalização com dificuldade } \\
\text { Barreira física (traQueostomia,intubação orotraQueal, máscara facial) } \\
\text { Condição fisiológica } \\
\text { Condição emocional }\end{array}$ & 28 \\
\hline
\end{tabular}

Das intervenções propostas pela NIC para o diagnóstico de enfermagem Risco para aspiração: controle das vias aéreas, monitorização respiratória e precauções contra aspiração, todas foram checadas pelas enfermeiras assistenciais da UTI Materna. A dificuldade de elevar a parte superior do corpo, a depressão dos reflexos da tosse e deglutição e o aumento da pressão intragástrica, podem levar à aspiração de secreções. Como mostra a experiência, as conseqüências dessa situação podem ser desastrosas para o paciente, pois a aspiração do conteúdo gástrico pode provocar irritação e destruição da mucosa traqueal e pneumonias, aumentando o risco de infecçãa ${ }^{(12)}$.

Das principais intervenções propostas pela NIC para o diagnóstico de enfermagem Padrão respiratório ineficaz: controle das vias aéreas e aspiração das vias aéreas, todas foram checadas pelas enfermeiras assistenciais da UTI Materna. Trata-se de um DE bastante freeüente em pacientes de UTI, ocasionado tanto pela patologia de base, como pela dor, ansiedade ou fadiga da musculatura respiratória.

Diante do panorama de intervenções propostas versus realizadas pelas enfermeiras da UTI Materna, percebe-se o empenho destas na prevenção de infecções, do deseQuilíbrio do volume de líquidos e da aspiração, assim como na manutenção da integridade da pele e promoção de um padrão respiratório eficaz.

Os diagnósticos, características definidoras e fatores relacionados 
do padrão RELACIONAR foram: interação social prejudicada $e$ maternidade alterada (ambos presentes em todos os casos).

Das principais intervenções propostas pela NIC para o diagnóstico de enfermagem Interação social prejudicada: aumento da socialização e jogo terapêutico, nenhuma foi checada pelas enfermeiras assistenciais, demonstrando uma lacuna no cuidado referente ao aumento do sistema de apoio e à reintegração social.

Das principais intervenções propostas pela NIC para o diagnóstico de enfermagem Maternidade alterada: promoção da maternidade, aumento do sistema de apoio, promoção do envolvimento familiar e processo de estabelecimento do vínculo mãe-bebê, nenhuma foi checada pelas enfermeiras assistenciais.

A partir do momento em Que as mulheres descobrem a gestação, elas passam a nutrir um novo papel perante o grupo social no Qual estão inseridas, o de ser mãe. Contudo, complicações obstétricas Que implicam hospitalização, irão prejudicar esse vínculo mãe-filho, contribuindo para uma situação desgastante e penosa não só para ambos, mas para toda a família. Em sua pesquisa, determinados autores $^{(13)}$ verificaram achado similar. Segundo afirmam, é válido o enfermeiro se instrumentalizar para auxiliar essa família a transpor esse momento de crise.

O diagnóstico, características definidoras e fatores relacionados do padrão MOVER foi mobilidade física prejudicada (presente em todos os casos).

Das principais intervenções propostas pela NIC para o diagnóstico de enfermagem Mobilidade física prejudicada: promoção de exercícios e mudança postural, todas foram checadas pelas enfermeiras assistenciais.

Em consonância com estudo realizado por alguns autores ${ }^{(14)}$, a mobilidade física prejudicada também esteve presente em 100\% dos participantes da pesquisa ora desenvolvida. Por ser esta uma condição de limitação do movimento físico do corpo ou de uma ou mais extremidades, pode acompanhar e/ou agravar outros diagnósticos de enfermagem já existentes, como risco para integridade da pele prejudicada, risco de infecção ou risco para desequilíbrio no volume de líquidos.

O diagnóstico, características definidoras e fatores relacionados do padrão SENTIR foi ansiedade (presente em todos os casos).

Das principais intervenções propostas pela NIC para o diagnóstico de enfermagem Ansiedade: assistência no controle da raiva, treinamento para o controle dos impulsos, melhora do enfrentamento, redução da ansiedade e oferecimento de apoio psicológico, apenas as duas últimas foram checadas pelas enfermeiras assistenciais.

Como é notório, a UTI representa um local estressante para as pacientes, Que vivenciam o medo da morte, além de um ambiente de constante tensão, com ruídos e luzes nas 24 horas do dia. Tudo isso contribui para aumentar a ansiedade.

O diagnóstico, características definidoras e fatores relacionados do padrão COMUNICAR foi Comunicação verbal prejudicada (presente em todos os casos).

Das principais intervenções propostas pela NIC para o diagnóstico de enfermagem Comunicação verbal prejudicada: melhora da comunicação, ouvir ativamente e incentivar a comunicação, todas foram checadas pelas enfermeiras assistenciais.

\section{CONSIDERAÇÕES FINAIS}

Como mostra o estudo, é evidente o Quanto a introdução do plano de cuidados em enfermagem na Unidade de Terapia Intensiva Materna possibilita melhorar a assistência obstétrica. À medida Que os componentes da equipe vão percorrendo todas as etapas do processo, desde a coleta da história do paciente, passando pelo julgamento clínico, formulação dos diagnósticos de enfermagem, elaboração e implementação das intervenções, eles irão desenvolver um cuidado individualizado capaz de atender às reais necessidades do cliente, além de demonstrar Qualidade em seu trabalho, conforme as metas forem alcançadas.

A adoção do processo de enfermagem como instrumento tecnológico ou modelo metodológico Que orienta a prática profissional assume características de prática reflexiva do enfermeiro sobre os significados implícitos em suas ações, reações e transações com a paciente. Desse modo, possibilita-lhe mudar seu raciocínio, julgamento e ações, transformando o modo de pensar para uma mudança na prática ${ }^{(15)}$.

A realização deste trabalho revelou-se significativa e propiciou às autoras sair um pouco da posição de enfermeira assistencial e visualizar a realidade de cada mulher internada em uma UTI materna, à luz de algumas etapas da metodologia da assistência em enfermagem.

Foi uma mudança de lugar necessária, pois a correria do diadia, muitas vezes, impede ver os pacientes de forma singular, mesmo no caso de profissionais Que procuram sempre dar o melhor de si. Inegavelmente, persistem lacunas no cuidado de enfermagem, sobretudo num ambiente de cuidados críticos, onde se encontram pacientes extremamente dependentes, com sua auto-estima abalada, e Quase sempre despersonificados e desesperançados.

Diante dos achados, sugere-se uma reflexão sobre o atendimento prestado às mulheres internadas em uma Unidade de Terapia Intensiva Materna, tanto no referente ao planejamento do cuidado, Quanto na sua coordenação, organização, execução e avaliação. Só assim se terá condições de implementar uma prática inovadora e Que reflita para todos, a médio ou a longo prazo, melhoria na Qualidade da assistência materna.

\section{REFERÊNCIAS}

I. Rezende J,Montenegro CAB. Obstetrícia fundamental. Rio de Janeiro: Guanabara Koogan; 2003.

2. Leopardi MT. Teorias de enfermagem: instrumentos para a prática. Florianópolis: Papa-Livros; 1999.

3. Potter PA, Perry AG. Fundamentos de enfermagem: conceitos, processo e prática. Rio de Janeiro: Guanabara Koogan; 2004.

4. Branden PS. Enfermagem materno infantil. Rio de Janeiro: Reichmann \& Affonso; 2000.

5. Risner PB. Nursing diagnosis: diagnostic sistements. In: Christensen PJ, Kenney IW. Nursing process: application of conceptual modes. $3^{\text {rd }}$ ed. St. Louis: Mosby; 1990.

6. Carvalho EC, Jesus CAC. Raciocínio clínico na formulação de 
diagnóstico de enfermagem para o indivíduo. Série didática: enfermagem no SUS. Brasília: Associação Brasileira de Enfermagem; 1997.

7. North American Nursing Diagnosis Association. Diagnósticos de enfermagem da NANDA: definições e classificações - 1999. 2000. Porto Alegre: Artes Médicas Sul; 2000.

8. Johnson M, Bulechek G, Dochterman IM, Maas M. Diagnósticos, resultados e intervenções de enfermagem - ligações entre NANDA, NOC e NIC. Porto Alegre: Artmed; 2005.

9. Ministério da Saúde (BR). Diretrizes e normas regulamentadoras da pesquisa envolvendo seres humanos. Brasília: Ministério da Saúde; 1997.

10. Freitas F, Martins SM, Ramos JGL. Rotinas em obstetrícia. Porto Alegre: Artmed; 2002.

I1. Pereira MS, Prado MA, Sousa IT, Tipple AFV, Souza ACS. Controle de infecção hospitalar em unidade de terapia intensivadesafios e perspectivas. Rev Elet Enfermagem [online] 2000 out;2(1):309-23. Disponível em: http://www.revistas.ufg.br/ index.php/fen

12. Galdeano LE, Rossi LA, Nobre LF, Ignácio DS. Diagnóstico de enfermagem de pacientes no período transoperatório de cirurgia cardíaca. Rev Lat-am Enfermagem 2003; I I (2): 760-3.

13. Vieira CS, Rossi LA. Os diagnósticos de enfermagem da taxonomia da NANDA em mulheres com o filho prematuro hospitalizado e o sistema conceitual de King. Rev Lat-am Enfermagem 2000; 8(6): 800-4.

14. Rocha LA, Maia TF, Silva LF. Diagnósticos de enfermagem em pacientes submetidos à cirurgia cardíaca. Rev. Bras Enferm 2006; 59(3):307-2.

15. Garcia TR, Nóbrega MML, Carvalho EC. Nursing processapplication to the professional practice. OBIN [online] 2004 jul; 3 (2): [7 screens]. Available from: http://www.uff.br/nepae/ objn302garciaetal.htm 